\title{
化学链钒氧载体及覆盖度效应研究
}

张涛

中国科学院大连化学物理研究所, 催化基础国家重点实验室, 辽宁大连 116023

\section{Vanadia Oxygen Carrier and Its Coverage Effect for Chemical Looping Processes}

\section{Tao Zhang}

State Key Laboratory of Catalysis, Dalian Institute of Chemical Physics, Chinese Academy of Sciences, Dalian 116023, Liaoning Province, China.

Email: taozhang@dicp.ac.cn

Published online: December 9, 2020.
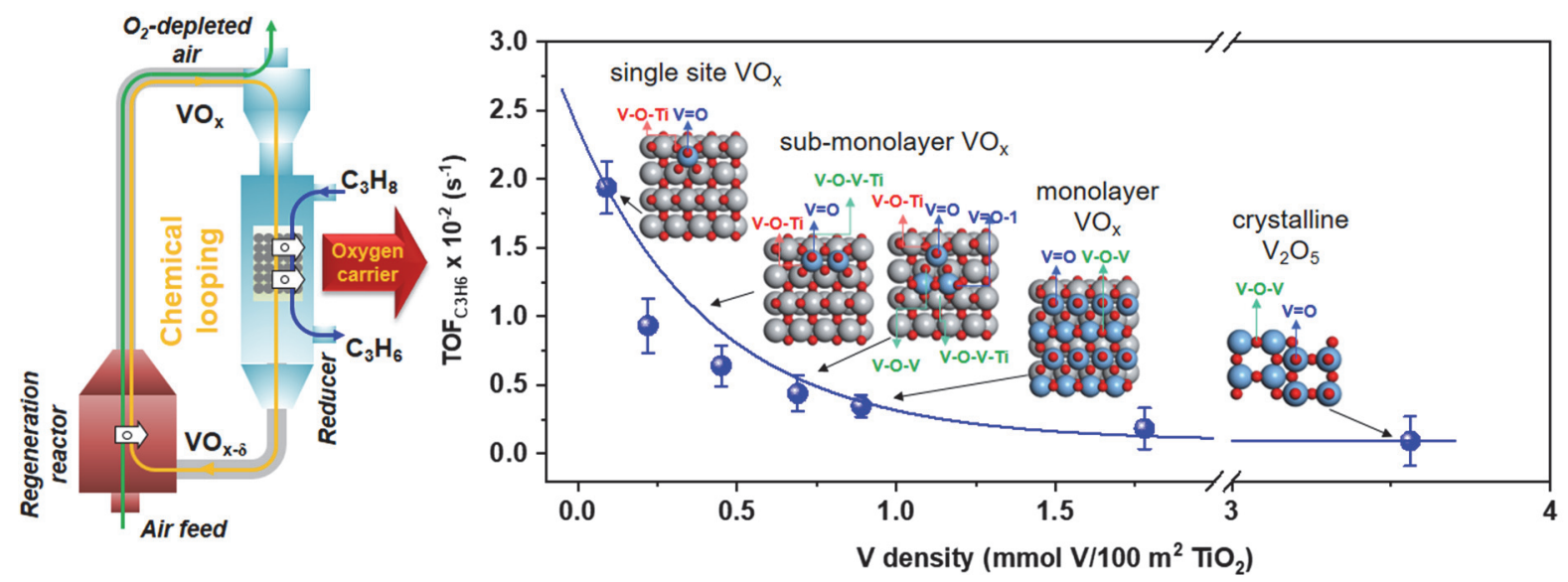

丙烷化学链氧化脱氢钒氧载体及其覆盖度效应。

丙烯是化工行业重要的基础原料之一。在众 多生产丙烯的工艺中, 丙烷无氧脱氢(PDH)以丙烯 选择性高、技术成熟度高、投资成本低的特点, 成 为目前最具竞争力的丙烯生产工艺 ${ }^{1,2}$ 。但商业化 的 $\mathrm{Pt}$ 基和 $\mathrm{Cr}$ 基催化剂因其成本高昂、环境污染和 丙烷转化率受热力学平衡限制等因素而影响了其 应用和发展 3,4 。因此, 开发新型、可替代的脱氢工 艺及与之配套的催化剂体系具有重要的科学研究 和工业应用价值。

化学链过程(chemical looping process)作为一 种新型的过程强化技术, 重新设计反应路径, 将总 反应分解为不同空间或时间内进行的两个或多个 子反应, 通过固态氧载体(通常为金属氧化物)在系
统中传递物质和能量 5 。天津大学巩金龙教授研究 组和俄亥俄州立大学范良士教授研究组指出化学 链氧化脱氢(CL-ODH) 是一种潜在的新型、可替代 的脱氢工艺 6 。通过固态氧载体材料, 可以将丙烷 脱氢分解为脱氢和再生两个子反应。氧载体在还 原床反应器中被丙烷还原, 接着回流到再生床反 应器中被再生, 完成氧化还原循环。2019年, 陈赛 等人构建了 $\mathrm{Mo}-\mathrm{V}-\mathrm{O}$ 混合钒氧载体体系, 实现了 $\mathrm{Mo}$ 原子级别的掺杂 7 。在 $500{ }^{\circ} \mathrm{C}$ 反应条件下, 获得 了 $35 \%$ 丙烷转化率和 $89 \%$ 丙烯选择性, 并且在 100 次氧化再生循环中保持稳定, 打破了同等测试条 件下丙烷无氧脱氢的热力学平衡收率。基于 $\mathrm{V}-\mathrm{O}$ 晶格氧物种识别和扩散行为的探究, 进一步揭示 
了动态晶格氧物种和产物选择性的相关性关系。

尽管研究者们在化学链氧载体的开发设计中 取得了一定进展, 然而, 由于 $\mathrm{V}-\mathrm{O}$ 物种的多样性 以及晶态氧化钒晶格氧体相扩散速率不均一, 导 致表面反应的活性位点不明晰。美国 Lehigh University 的 Israel E. Wachs研究组早前研究表 明, 在有氧气参与的丙烷氧化脱氢反应中, $\mathrm{V}-\mathrm{O}-$ 载体位点(既不是 $V-O-V$, 也不是末端 $V=O$ 位点) 似乎是关键活性位点 8 。然而, 在化学链氧化脱氢 反应体系中, 随着晶格氧的逐渐消耗与晶相结构 的动态演变, 氧化钒氧载体表面位点和反应活性 的关系, 目前尚不清楚。

为了明确化学链反应过程钒氧载体的活性位 点, 天津大学巩金龙教授课题组采用浸渍法, 通过 调节氧化钒负载量, 在金红石 $\mathrm{TiO}_{2}$ 载体上构建了 规整的亚单层或单层氧化钒纳米结构。基于拉曼 光谱(Raman)和高倍透射电镜(HR-TEM) 分析, 氧 化钒在 $\mathrm{TiO}_{2}$ 载体上以孤立、聚合和晶态形式存在, 且其形式和负载量存在对应关系。当氧化钒负载 量远远低于单层分散阈值时 $(\sim 1 \%(w))$, 氧化钒物 种主要由孤立的氧化钒通过界面的 $\mathrm{V}-\mathrm{O}-\mathrm{T}$ 键桥 接到 $\mathrm{TiO}_{2}$ 载体表面。随着氧化钒负载量的进一步 增加, 孤立的氧化钒物种通过 $\mathrm{V}-\mathrm{O}-\mathrm{V}$ 键连接形 成聚合态的氧化钒物种。而当氧化钒负载量超过 单层分散的阈值时, 晶态 $\mathrm{V}_{2} \mathrm{O}_{5}$ 形成。

进一步, 将其用于丙烷脱氢反应, 亚单层或单 层的氧化钒获得了近乎 $90 \%$ 的丙烯选择性, 高于晶 态的 $\mathrm{V}_{2} \mathrm{O}_{5}$ 氧载体, 并且丙烯转换频率(TOF)随氧化 钒负载量的增加而减小。进一步借助程序升温还 原 $\left(\mathrm{H}_{2}-\mathrm{TPR}\right)$ 、程序升温表面反应 $\left(\mathrm{C}_{3} \mathrm{H}_{8}-\mathrm{TPSR}\right)$ 和原 位拉曼光谱( in situ Raman) 分析, 发现丙烷脱氢活 性和氧化钒-氧化钛界面作用密切相关。具体来 说, 随着氧化钒负载量的增加, 界面 $\mathrm{V}-\mathrm{O}-\mathrm{Ti}$ 与 $\mathrm{V}-\mathrm{O}-\mathrm{V}$ 键的比值降低, 导致晶格氧反应活性降 低, 从而使反应TOF降低。而当氧化钒负载量高于
单层分散的阈值, 晶态 $\mathrm{V}_{2} \mathrm{O}_{5}$ 中晶格氧扩散到表面 演变成亲电氧物种, 导致了丙烯过度氧化和丙烯 选择性的降低。其实验结果与密度泛函理论计算 结果非常吻合。

上述研究工作近期在 Angewandte Chemie International Edition 上以热点论文形式在线发 表 ${ }^{9}$ 。此项工作建立了化学链氧载体覆盖度与表面 反应的相关性关系, 明确了氧载体表面氧物种与 反应性能的构效关系, 完善了晶格氧动态扩散机 制, 建立了晶格氧调控反应动力学的新方法, 为高 性能氧载体开发和烷烃脱氢奠定了理论基础和提 供了新的研究思路。

\section{References}

(1) Chen, S.; Pei, C.; Sun, G.; Zhao, Z.-J.; Gong, J. Acc. Mater. Res. 2020, 1, 30. doi: 10.1021/accountsmr.0c00012

(2) Otroshchenko, T.; Jiang, G.; Kondratenko,V. A.; Rodemerck, U.; Kondratenko, E. V. Chem. Soc. Rev. 2020, doi: 10.1039/d0cs01140a

(3) Chen, S.; Zhao, Z.-J.; Mu, R.; Chang, X.; Luo, J.; Purdy, S. C.; Kropf, J. A.; Sun, G.; Pei, C.; Miller, J. T.; et al. Chem 2020, doi: 10.1016/j.chempr.2020.10.008

(4) Sattler, J. J. H. B.; Ruiz-Martinez, J.; Santillan-Jimenez, E.; Weckhuysen, B. M. Chem. Rev. 2014, 114, 10613. doi: $10.1021 / \mathrm{cr} 5002436$

(5) Zhu, X.; Imtiaz, Q.; Donat, F.; Müller, C. R; Li F. Energy Environ. Sci. 2020, 13, 772. doi: 10.1039/C9EE03793D

(6) Zeng, L.; Cheng, Z.; Fan, J. A.; Fan, L.-S; Gong, J. Nat. Rev. Chem. 2018, 2, 349. doi: 10.1038/s41570-018-0046-2

(7) Chen, S.; Zeng, L.; Mu, R.; Xiong, C.; Zhao, Z.-J.; Zhao, C.; Pei, C.; Peng, L.; Luo, J.; Fan, L.-S.; Gong, J. J. Am. Chem. Soc. 2019, 141, 18653. doi: $10.1021 /$ jacs.9b09235

(8) Wachs, I. E.; Weckhuysen, B. M. Appl. Catal. A 1997, 157, 67. doi: 10.1016/S0926-860X(97)00021-5

(9) Chen, S.; Pei, C.; Chang, X.; Zhao, Z.-J.; Mu, R.; Xu, Y.; Gong, J. Angew. Chem. Int. Ed. 2020, 59, 22072. doi: 10.1002/anie.202005968 